\title{
Synoptic Analysis of Epidemiologic Evidence of Brain Cancer Risks from Mobile Communication
}

\author{
Norbert Leitgeb \\ Institute of Health Care Engineering with European Notified Body of Medical Devices, Graz University of \\ Technology, Graz, Austria \\ Email: norbert.leitgeb@tugraz.at
}

Received 2 September 2014; revised 1 October 2014; accepted 27 October 2014

Copyright (C) 2014 by author and Scientific Research Publishing Inc.

This work is licensed under the Creative Commons Attribution International License (CC BY). http://creativecommons.org/licenses/by/4.0/

(c) (i) Open Access

\begin{abstract}
In the radio frequency (RF) range concern about long-term health risks from electromagnetic fields (EMF) is enhanced by contradictory results and conclusions from epidemiologic studies. A new approach of a synoptic analysis of all available data from epidemiological studies published since 2001 was performed. This approach provided new insight with regard to a potential link between mobile phone use and brain cancer. Two quite different data pools could be identified with numerous studies from one research group opposing all other studies. However, it could be shown that with the number of exposed cases both data pools exhibit a clear trend of risk estimates (odds ratios) towards the final result, namely a reduced cancer risk of $\mathrm{OR}=0.8$, though from either side of the zero-risk line. The analysis of potential long-term effects indicated by a dosedependence revealed diverging results with different dose metrics. Overall, the synoptic analysis supports reassuring rather than alarming conclusions on RF EMF health risks from mobile telecommunication.
\end{abstract}

\section{Keywords}

Health Risk, Long-Term Effect, Carcinogenicity, Electromagnetic Field, Mobile Phone

\section{Introduction}

In the past, already several times technological progress has dramatically changed the electromagnetic environment, such as the start of wide-spread use of electricity at the end of the $19^{\text {th }}$ century which made extremely low (power) frequency (ELF) electric fields (EF) and ELF magnetic fields (MF) omnipresent in daily life. Then 
broadcasting made radio frequency (RF) electromagnetic fields (EMF) up to about $100 \mathrm{MHz}$ appearing throughout countries, worldwide and later television added RF EMF of several $100 \mathrm{MHz}$. Therefore, the introduction of mobile telephony with worldwide dissemination which added to or replaced former analogue technology with frequencies around $1 \mathrm{GHz}$ is not a unique event with regard to changes in EMF environment.

In the meanwhile, ELF MF and RF EMF became a source of public concern about potentially induced adverse health effects, including cancer. This triggered considerable scientific effort to clarify whether or not there might be a health risk even at levels well below existing exposure limits. Similar to the ELF range, in spite of meanwhile numerous studies, also in the RF range public concern is still increasing as indicated by the continuously increasing number of publications related to RF EMF health risk. This could be demonstrated by a literature search in the Google Scholar database (Figure 1).

Based on epidemiologic evidence for a possible association of ELF MF with childhood leukemia which was concluded to be limited, the International Agency for Research on Cancer (IARC) of the World Health Organization (WHO) has classified MF of the entire ELF range as possibly carcinogenic to humans class 2B [1]. Likewise, again mainly based on limited epidemiologic evidence IARC classified EMF of the entire RF range as possibly carcinogenic to humans class 2B [2].

Existing recommendations, standards or laws aim at preventing the population from any established adverse health effect from RF EMF [3]-[5]. However, the regulations still include disclaimers that exposure limits would not account for potential long-term health effects. As an example, in its EMF directive for workers the European Commission stated that the directive "did not address the long-term-effects, including the possible carcinogenic effects, of exposure to time-varying electric, magnetic and electromagnetic fields, for which there is currently non conclusive scientific evidence establishing a causal relationship" which is a wording that suggests such effects could be expected in the future [4]. It is obvious that such a wording is not extremely reassuring.

In the ELF range concern is driven by contradictory results of individual studies and the fact that the body of epidemiologic studies including meta-analyses exhibits an offset towards an increased risk (solely) for childhood leukemia. However, the synoptic analysis of all available evidence could already reveal that, overall, with increasing numbers of exposed cases included in a study the evidence for childhood leukemia convincingly approaches the zero risk line and finally stays there [6].

However, in the RF range the situation regarding epidemiologic studies is somewhat more difficult. It is characterized by results and risk assessment from a particular group opposing all other studies. On the one hand, numerous studies of the Swedish group of Hardell et al. (SHG) report on consistent and statistical significantly elevated risk of brain cancer [7]-[14]. On the other hand, there are results of the multinational interphone study (IPS) with 13 countries participating [15] [16], and numerous other national studies (ONS) including Sweden which report on a lack of carcinogenic evidence up to 10 years mobile phone use with some uncertainty with regard to specific cancers at prolonged exposure [17]-[36].

These two subgroups of studies with each of them relying on their own consistent results contradictorily conclude on alarming as well as reassuring evidence leaving it up the public to choose whom to believe.

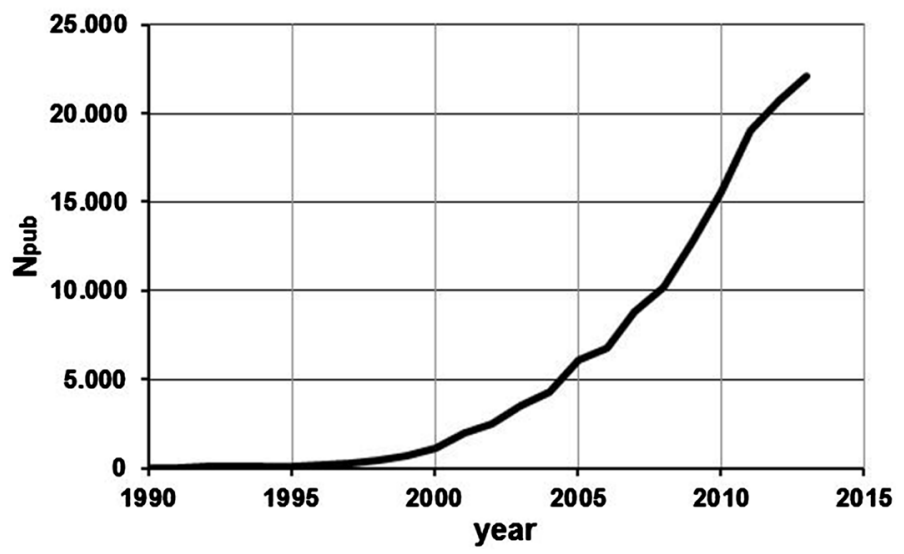

Figure 1. Number of publications $\mathrm{N}_{\text {pub }}$ listed by Google Scholar for the key words "EMF and health risk and RF electromagnetic fields". 
In this situation this paper presents a new approach by assessing the entire available evidence from epidemiological case-control studies and performing a synoptic analysis of all available data published so far rather than just relying on a few selected studies or some particular results picked out of the pool of evidence for drawing conclusions. It is shown that this new approach allows gaining new insights and contributes to health risk assessment.

\section{Method}

In the meanwhile it has become common practice that papers on epidemiologic studies include tables with an extensive number of detailed results in terms of risk estimates (odds ratios, OR) which have been calculated for a large variety of different scenarios such as kind of tumor, tumor location, laterality, radiation source (e.g. mobile phone, wireless, DECT), exposure scenario (e.g. urban or rural), magnitude of exposure and dose metric (e.g. cumulated number of calls, cumulated call time, subscription time, use years). However, so far conclusions are drawn by authors based on only one or few selected worst case OR values, likewise, health risk assessment bodies pick out such single values. So far, no use is made of the entire pool of data.

In the new synoptic approach all available ORs of available epidemiologic case-control studies published from 2001 until 2014 were presented and analyzed such as to identify any dose dependence. Studies were identified by a literature search in the data bases PubMed, FEMU and IEEE. In case values were given both unadjusted and adjusted for cofactors, the latter were used. The studies were included without further selection criteria such as the size of the study or exposure metric. The entire body of available data was analyzed without any further restrictions.

The pool of data was analyzed to check the following issues:

1) Is there a time-trend of the published data which could indicate substantial changes in design of epidemiologic studies which could impair the relevance of older data or a trend to any stable result? Therefore, the time dependence of reported risk estimates was analyzed.

2) Since the power of a study increases with its size, it could be expected that larger studies including pooled-analyses should result in more reliable risk estimates than smaller ones. Therefore, the body of data was analyzed in dependence on the number of exposed cases.

3) Different studies used quite different cut-off points for classifying exposure and dose, making their result difficult to compare. Therefore, the data were synoptically analyzed in dependence on quantitative dose metrics to identify a potential dose-response as an indicator for a causal relationship between RF EMF exposure and brain cancer.

\section{Results}

Overall, 30 epidemiologic studies on mobile phone use and brain cancer with 2211 reported OR values could be analyzed [7]-[36]. Figure 2 presents an overview on the pooled data related to overall or selected brain tumors irrespective their statistical significance. It shows the dependence on the number of exposed cases $\mathrm{N}_{\text {exp }}$ starting with ORs with very weak power since they had been calculated even on the basis of one exposed case only. Overall, the data exhibit a pronounced dependence on $\mathrm{N}_{\text {exp }}$ in terms of a funnel-shaped pattern with a wide variance of ORs at low $\mathrm{N}_{\text {exp }}$ extending from 0.18 up to 8.10 at either side of the zero risk line $\mathrm{OR}=1$. With increasing $\mathrm{N}_{\mathrm{exp}}$ the variance decreases. However, it can be seen that data exhibit two separate clusters. This confirms the systematic differences between reported results of the SHG and all other existing studies (AOS) which include ANS and IPS. It needs to be noted that the data of ANS and IPS exhibit a good overall agreement among each other. In contrast, it can be seen that SHG cluster is considerably shifted towards enhanced risk while the majority of AOD data are shifted towards the opposite side indicating a reduced cancer risk. It needs to be stressed that apart from the systematic differences, both data sets exhibit a strong dependency of reported OR values on $\mathrm{N}_{\text {exp }}$ although with differences in the details. It should be noted, that ORs tend towards reduced risk from either side of the zero-risk line. This is demonstrated even clearer by the subset of statistic significant ORs as presented in Figure 5.

To identify potential reasons for the obvious discrepancy between SHG and AOS data as a parameter of the study design the ratio of the number of controls $\mathrm{N}_{\text {contr }}$ and the number of exposed cases $\mathrm{N}_{\exp }$ was analyzed. The synoptic analysis revealed that ORs do not depend only on $\mathrm{N}_{\text {exp }}$ as shown in Figure 2 but also on the associated number of controls $\mathrm{N}_{\text {contr }}$ as expressed by the ratio. It can be seen that in the IPS the highest ORs are found, 


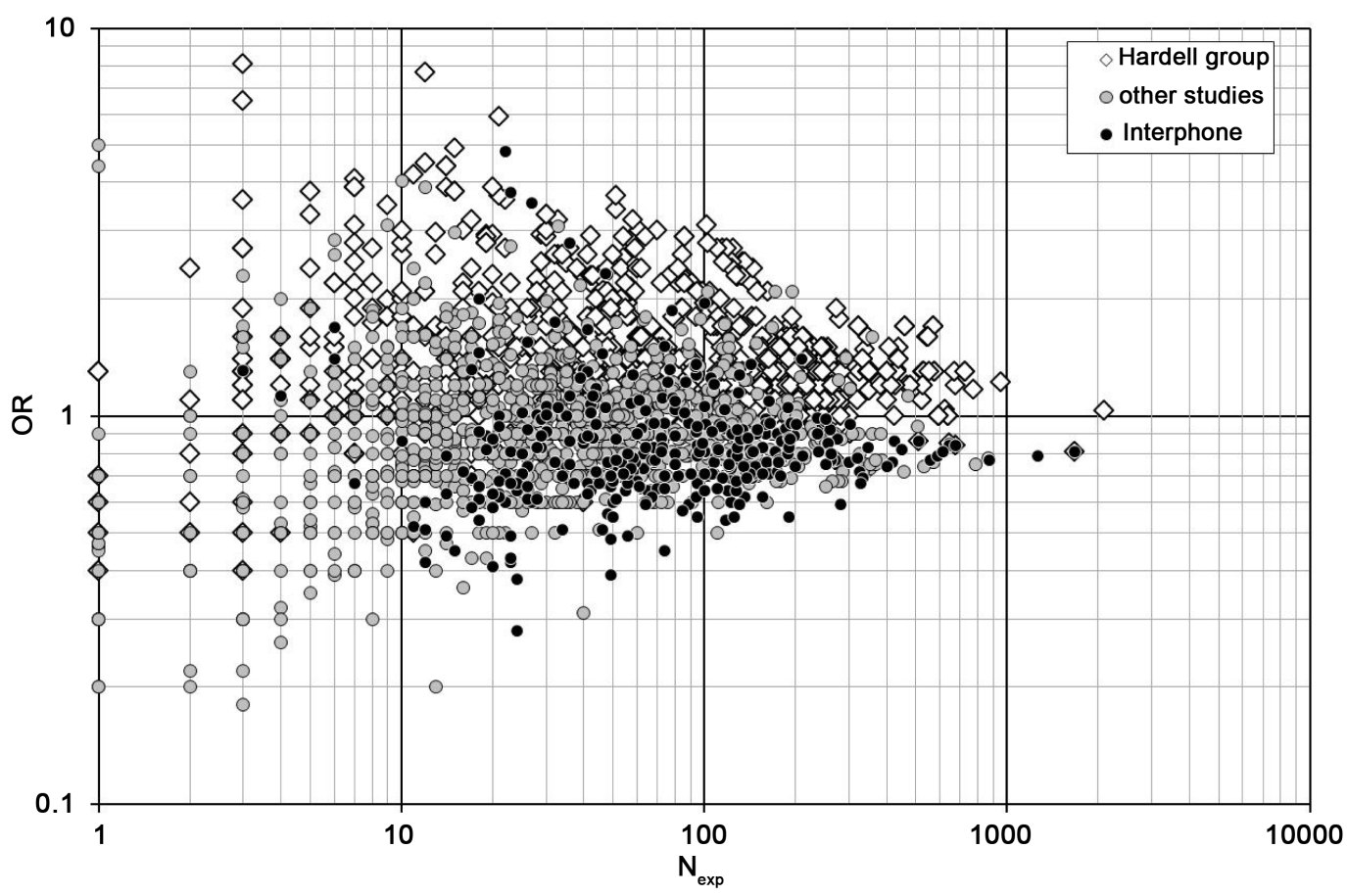

Figure 2. Reported odds ratios (OR) of epidemiological case-control studies on brain cancer and telecommunication handset use over the total number of exposed cases $\left(\mathrm{N}_{\exp }\right)$.

if $\mathrm{N}_{\text {contr }}$ was much lower than $\mathrm{N}_{\text {exp. }}$. In SHG data similar relationships can only be found in subsets. However, it needs to be noted that the ratio varies over a large span although study designs aimed at selecting at least as many controls as cases (Figure 3). The presentation demonstrates a striking difference between SHG and AOD also in respect to this parameter which is associated with the design of the study or at least with the design of the data analysis. In the IPS the range of controls per case extends over one order of magnitude from 0.22 to 3.44 . However, the situation is quite different with SHG data. In this case the range of controls per case is spread 100 fold wider and amounts to three orders of magnitudes, ranging from 0.14 to 126 . This also indicates a striking difference in the study design of SHG and IPS.

Because of the systematic difference of SHG and AOS data, time trend analysis had to be performed separately. SHG data showed only a slight increase of OR with time. The regression line remains above $\mathrm{OR}=1$ with a regression coefficient $R^{2}=0.0105$. Similar to this, the regression line of AOS data exhibits also a slight increase with a regression coefficient $\mathrm{R}^{2}=0.0114$ (Figure 4), however, at the opposite side of the zero-risk line.

In addition to Figure 1 the difference between SHG and AOS data becomes even clearer if the presentation is restricted to statistical significant ORs only. Figure 5 demonstrates the striking fact that the 8 SHG studies reported many more significant ORs than all the 31 other studies taken together. In addition, strikingly, all significant ORs reported by the SHG indicate increased risks with only one exception at a large $\mathrm{N}_{\text {exp }}$ which is $\mathrm{OR}=$ 0.8. It is interesting to note that SHG risk estimates exhibit a strong dependence on $\mathrm{N}_{\exp }$ in terms of a decreasing ORs with increasing $\mathrm{N}_{\text {exp }}$ towards zero risk and even beyond it. However, while there is some variance among the SHG data a puzzling cutoff can be seen at about $\mathrm{OR}=1.3$ which may indicate an artifact. However, there is still the lonesome value at the largest $\mathrm{N}_{\exp }$ which, interestingly, fits exactly with the result of AOS at this $\mathrm{N}_{\exp }$. The overall trend of SHG data to decrease with $\mathrm{N}_{\text {exp }}$ heads towards $\mathrm{OR}=0.8$. Apart from the overall pattern Figure 5 also demonstrates that statistical significant SHG results differ considerably from AOS data also in another aspect.

While almost all significant SHG results are well above OR $=1$ indicating enhanced risks, the majority of significant AOS data remains right at the opposite side of the zero-risk line $\mathrm{OR}=1$, hence, indicating a decreased cancer risk. It must be noted, however, that there are also AOS data above the zero-risk line which fit to the SHG data but clearly converge towards the limit value $\mathrm{OR}=0.8$. Interestingly, with increasing $\mathrm{N}_{\text {exp }}$ all statistical significant data both of SHG and AOS exhibit a conclusive trend of heading towards the risk estimate $\mathrm{OR}=0.8$. 


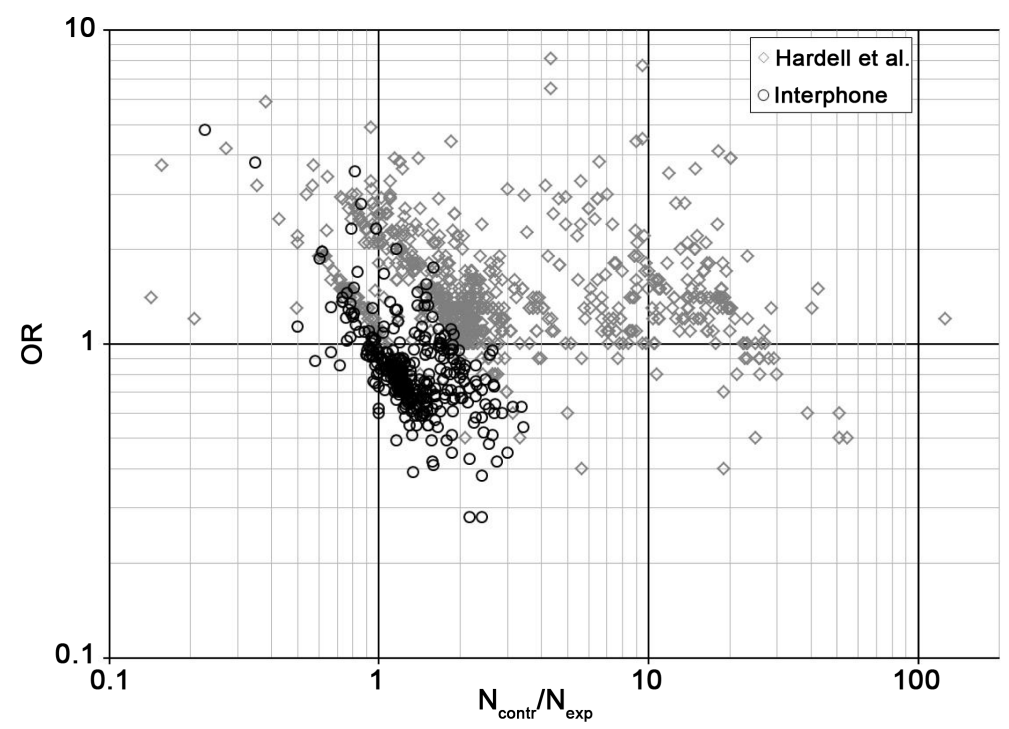

Figure 3. Reported odds ratios (OR) of epidemiological case-control studies on brain cancer and telecommunication handset use over the ratio of the number of controls $\left(\mathrm{N}_{\text {contr }}\right)$ and number of exposed cases $\left(\mathrm{N}_{\text {exp }}\right)$.
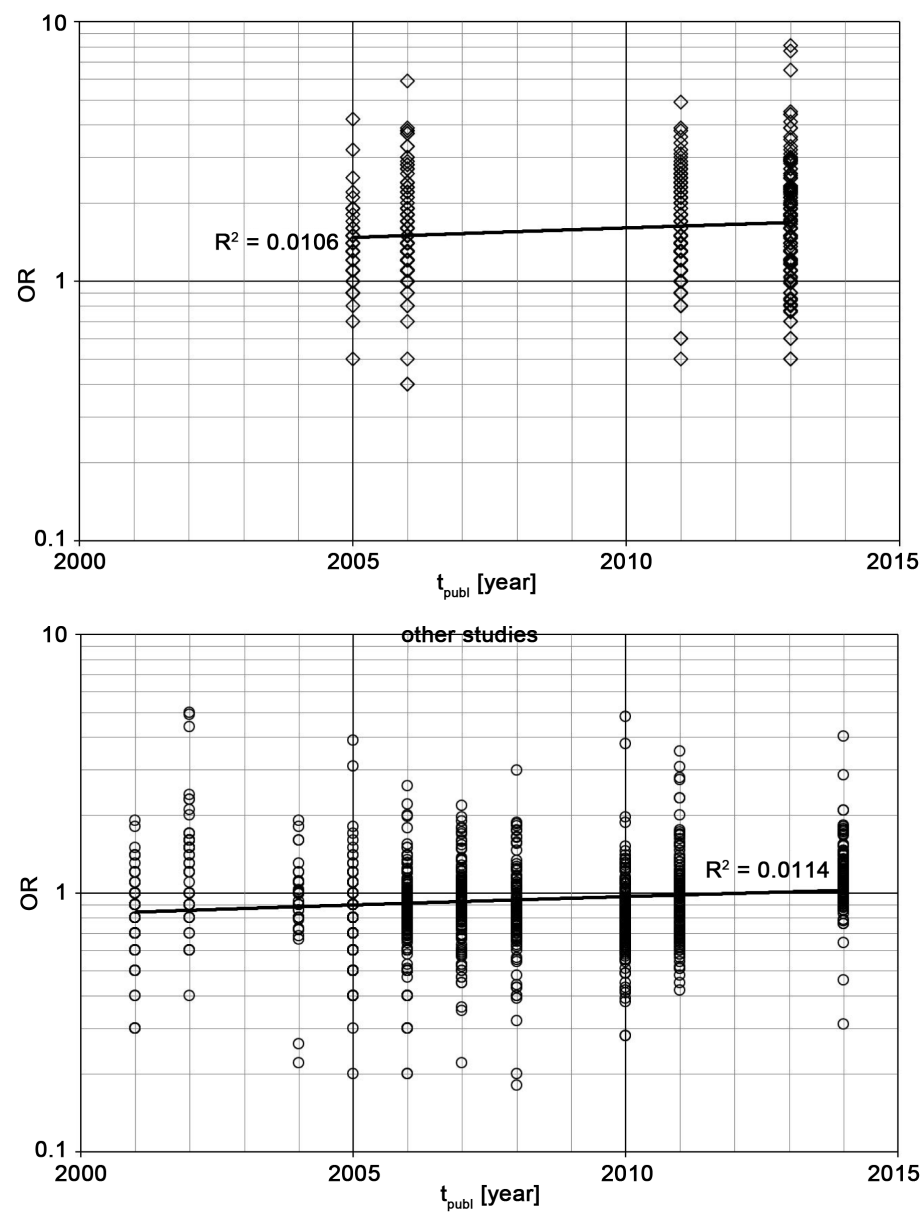

Figure 4. Reported odds ratios (OR) of epidemiological case-control studies on brain cancer and telecommunication handset use in dependence on year of publication (above: HDG data; below: AOS data, full circles... Interphone studies, open circles... other national studies except HDG). 


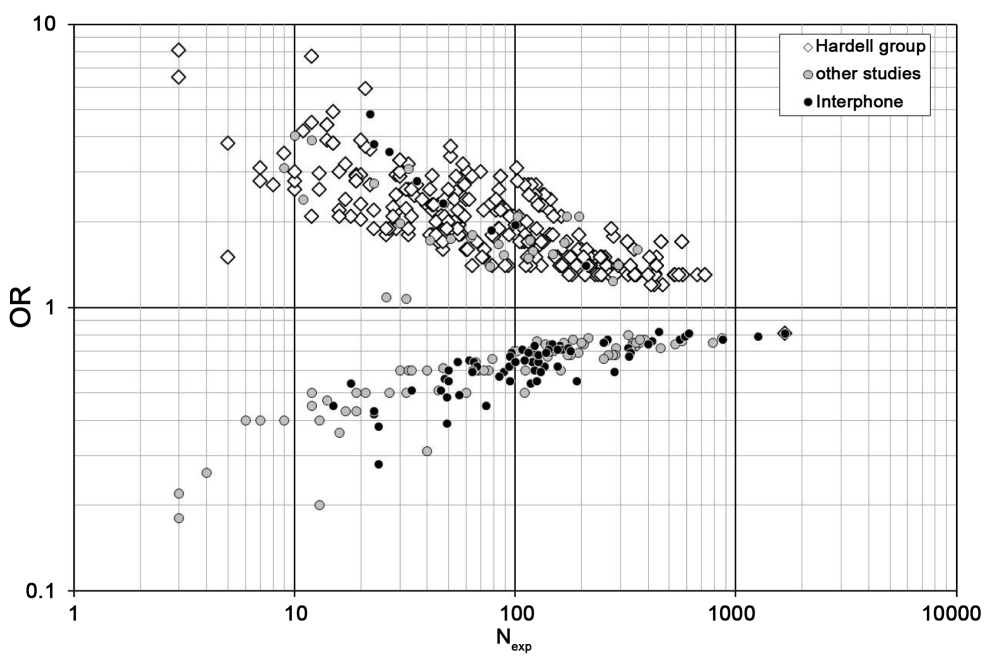

Figure 5. Odds ratios (OR) of epidemiological case-control studies on brain cancer and telecommunication handset use over the year of publication $t_{\text {publ }}$ (above: HDG data; below: AOS data).

In addition, the data were analyzed in dependence on dose. However, quite different results were found with different dose parameters which have been calculated based on guesses in questionnaires filled out by phone users and/or from subscription data of network providers.

With regard to the cumulated call time as derived from customer's estimates on average call duration, average number of calls and average use time per call (CCT) SHD data exhibit ORs which initially remain constant at the increased risk estimate OR $=1.5$ until a CCT of several $100 \mathrm{~h}$ and continuously increases afterwards (Figure 6). The quadratic regression coefficient amounts to $\mathrm{R}^{2}=0.2048$. Interestingly, data from AOS show a somewhat similar relationship, although at another OR level. Until a CCT up to several $100 \mathrm{~h}$ the data show no dose dependence, however, this time remaining at the level $\mathrm{OR}=0.8$. Only afterwards the regression curve continuously increases towards values larger than 1 . The quadratic regression factor is $\mathrm{R}^{2}=0.2867$ (Figure 6).

With regard to cumulated use years (CUY) as derived from questionnaires in terms of years of use or latency in terms of time of use prior diagnosis results are controversial between SHG and AOS (Figure 7). The quadratic regression curve of SHD data starts with a continuous increase of OR with CUY. The coefficient of quadratic regression amounts to $\mathrm{R}^{2}=0.1734$. In contrast to this, the analysis of AOS data shows a regression curve starting at zero risk towards decreased risk until about 5 CUY, and afterwards approaches again the zero risk value $\mathrm{OR}=1$. The regression coefficient of quadratic regression is $\mathrm{R}^{2}=0.0027$.

With regard to the dose metric number of calls (NOC) as derived from questionnaires, no data were available from SHG. Again the data from AOS exhibited almost no dependence on dose (Figure 8). The regression line remained flat over almost four orders of magnitudes at a reduced risk level of OR $=0.75$ and only afterwards slightly increases towards zero risk. The regression coefficient $\mathrm{R}^{2}=0.0283$.

\section{Discussion}

Instead of just picking out selected single results the synoptic analysis makes use of all existing evidence in terms of available data of epidemiologic case-control studies. This allowed identification of yet unknown quantitative relationships. Pooling data irrespective their statistical significance is justified by the fact that in the absence of any Bonferroni correction for multiparametric testing statistical significance of results of these studies might be challenged anyway. The synoptic analysis showed that also statistical non-significant ORs provide a valuable contribution. The patterns of the entire data allow gaining additional information and new insights.

The most interesting result is the strong dependence of ORs on $N_{\exp }$ which is a surrogate for the statistical power of a study. This is not self-evident and needs to be noticed. Such a dependence is not new. Interestingly, a similar overall pattern has been found with the data of ELF MF and childhood leukemia [6], however, now in case of RF EMF with two important differences:

Firstly, in the ELF range, the results of all published studies fitted well into one overall pattern. However, in 

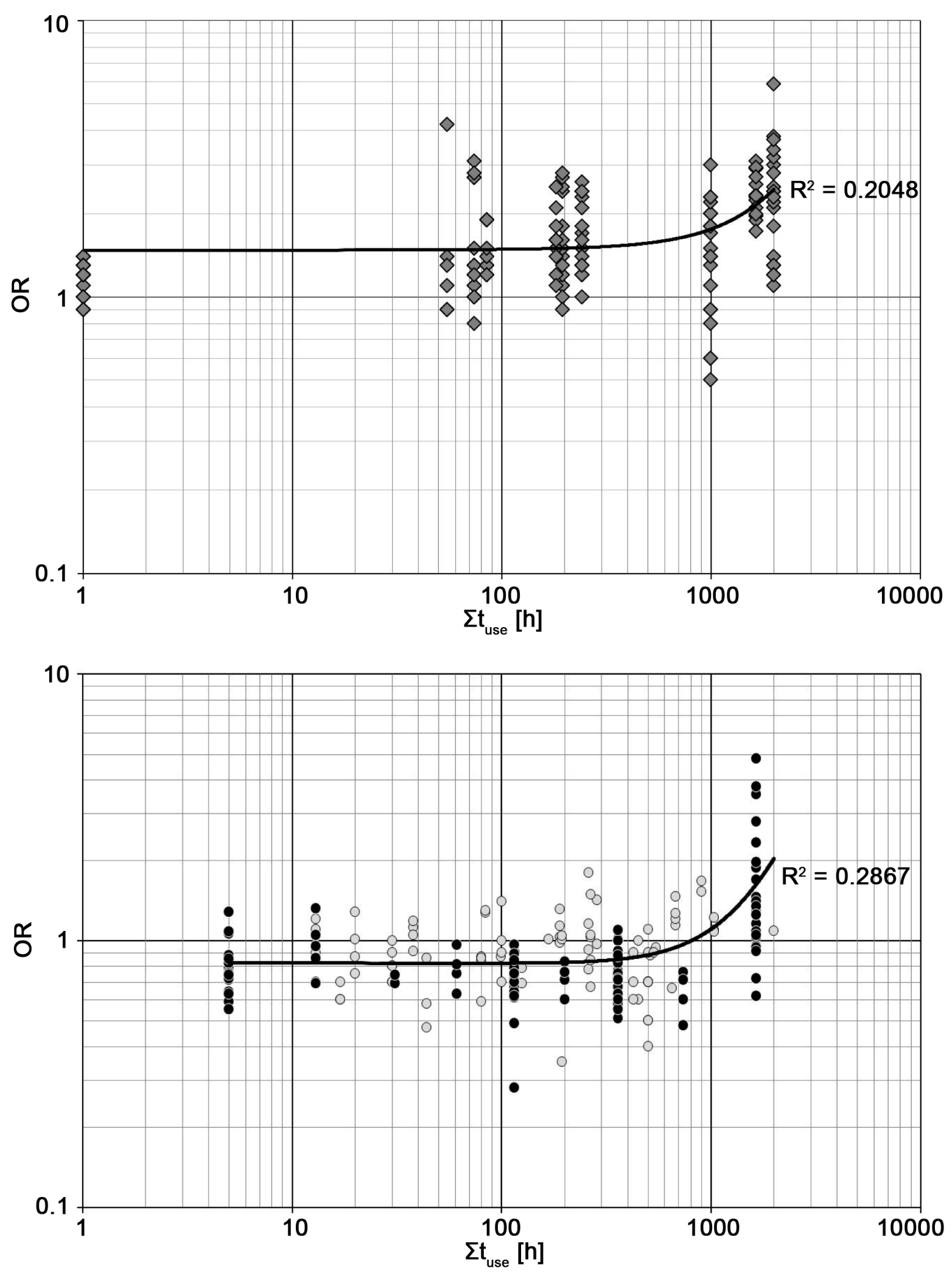

Figure 6. Reported odds ratios (OR) of epidemiological case-control studies on brain cancer and telecommunication handset use in dependence on the low border of cumulated call time intervals of exposure groups (above: HDG data; below: AOS data, full circles... Interphone studies, open circles... other national studies except HDG). 

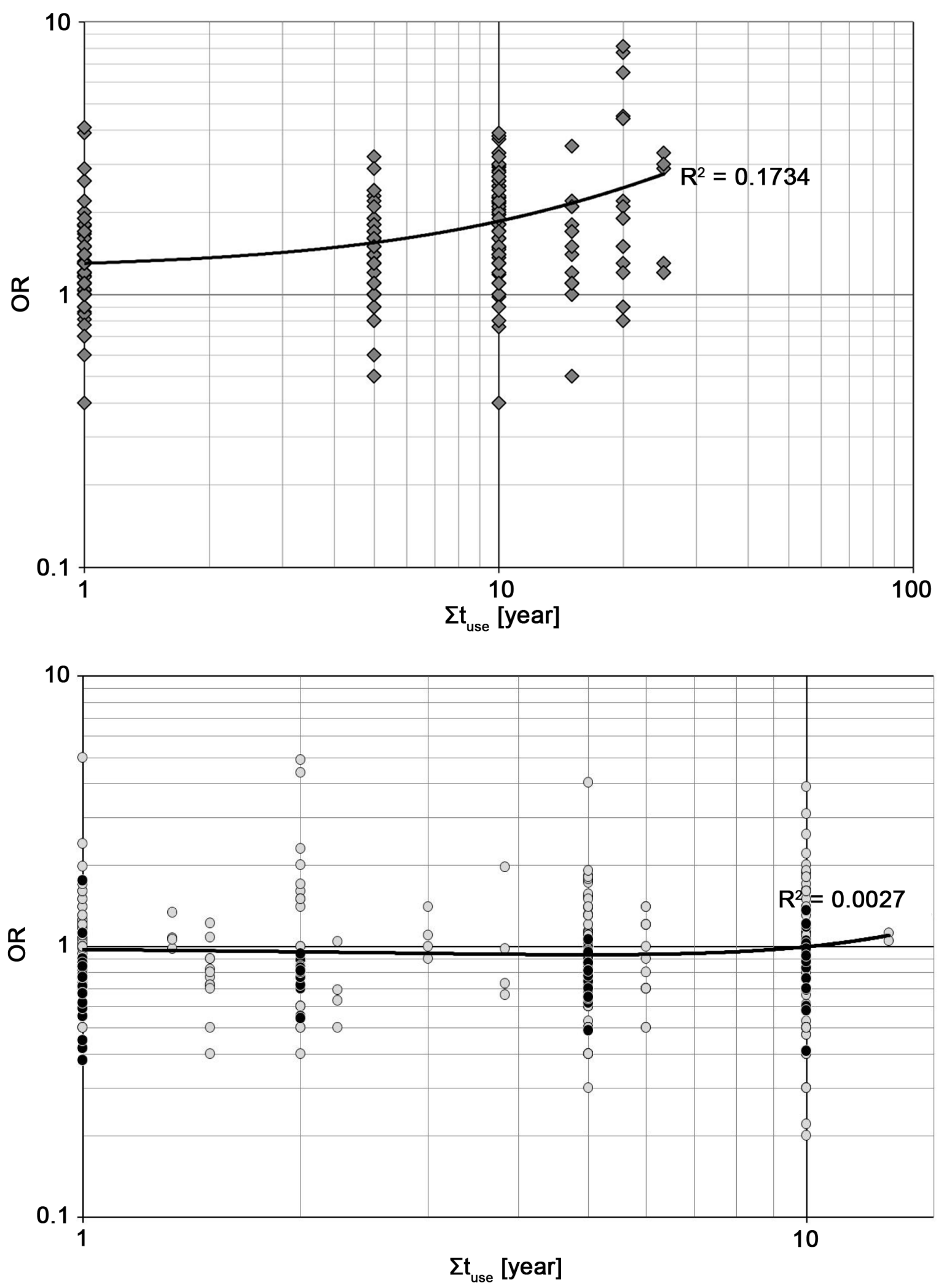

Figure 7. Reported odds ratios (OR) of epidemiological case-control studies on brain cancer and telecommunication handset use in dependence on the low border of cumulated use time intervals of exposure groups (above: HDG data; below: AOS data). 


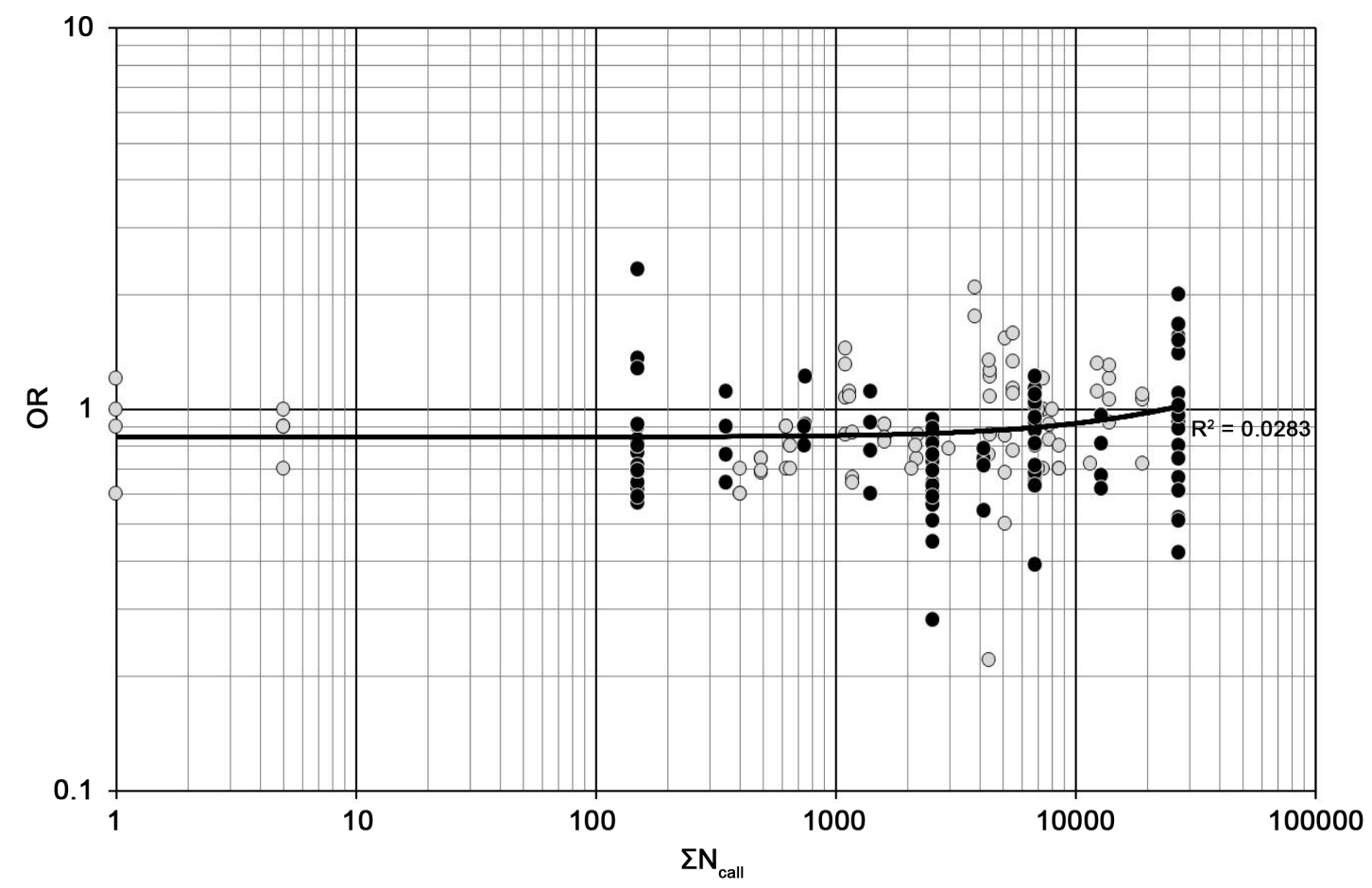

Figure 8. Reported odds ratios (OR) of epidemiological case-control studies on brain cancer and telecommunication handset use in dependence on the low border of cumulated number of calls intervals of exposure groups (AOS data).

contrast, the available RF data pool showed two quite different clusters with SHG data being systematically different in many aspects from all other published data and even other results based on the same Swedish population [18] [29] [31] [33]. Such a difference was already obvious just from the differences in the reported selected OR values. This has already been discussed by others [37] [38]. However, the presented synoptic analysis could reveal systematic deviations in the HDG study design which might help explain the encountered differences.

Secondly, in the ELF range with increasing $\mathrm{N}_{\exp }$ the ORs clearly and conclusively headed towards zero risk $\mathrm{OR}=1$, and above a certain $\mathrm{N}_{\exp }$ stayed there. In contrast to this, although with a similar trend, $\mathrm{RF}$ data tended towards a risk value associated with reduced risk. It needs to be stressed that this is found irrespective the puzzling differences between SHG and AOS data. But in contrast to the ELF case thanks the outmost right SHG data point, both data pools head towards the same risk estimate $\mathrm{OR}=0.8$, although from different sides of the zero-risk line. Consequently, both data pools agree in indicating a reduced cancer risk.

It is an important finding that the look at the entire data pool and the overall convergence at the end remove the discrepancy in the conclusions so far drawn from the SHG and AOD data. This could help overcome controversial debates.

However, the finding that in contrast to the ELF range, pooled RF ORs converge towards a reduced risk does not yet indicate that in fact RF EMF might be associated with cancer risk reduction. One major difference to the ELF range is, that in the RF range exposure assessment is a much greater challenge. This is due to the problem of gaining reliable quantitative data or adequate surrogates to estimate the local exposure from telecommunication headsets. In the ELF range exposure assessment is straighter forward because of a whole-body exposure and the known characteristics of MF field sources and their generated MF, while in the RF range exposure is local and its assessment has to rely on soft data which are prone to bias such as people's memory or subscriber data from network providers in particular with regard to historical exposure. To make it even more complicated exposure assessment has to cope with frequent changes in mobile telecommunication technology, devices and use pattern.

However, pooling of the entire data includes all exposed groups irrespective of the magnitude and/or duration of exposure. Consequently, it could be argued that groups of weakly exposed persons could have a potential lower risk which could mask a long-term effect in terms of a higher risk at higher doses. Of course, this hypo- 
thesis does not explain the encountered risk reduction. However, to consider this argument a potential dependence on dose was studied. The results showed that different exposure metrics exhibited quite different dose responses. From AOS data a long-term risk could hardly be concluded from cumulated call numbers and cumulated use years. However, with cumulated call time the result differed. While there was no dose response up to several hundred hours, at larger CCT a delayed increase of ORs could be found towards OR $=1$ indicating either a delayed onset of a long-term effect or an artifact. It is not yet clear whether CCT is a more reliable dose metric than CUY or CCN and whether it is indeed associated with a long-term effect.

There are several indications supporting an artifact hypothesis such as

a) the CCT results are not supported by those of other dose parameters. It needs to be noted that the quantitative relationship among the various dose parameters and with regard to tissue exposure is yet unknown;

b) the assessment of the largest exposure period suffers from the highest uncertainty;

c) a delayed dose-response would be in contradiction to existing well established knowledge on physical and biological interaction mechanisms;

d) there is no interaction model neither physical nor biological which could explain such a dose-dependence;

e) a delayed dose-response would necessarily have to result from a crossover effect from one call to another which needs to last long enough to bridge the gap of even many hours or days to allow a cumulative effect be generated;

f) there is no explanation why potential crossover effects, if existent would not continuously cumulate with time but could lead to a biologic response delayed by many years;

g) a delayed dose-response would require the long-term absence of any repair mechanism for potential effects.

Therefore, whether the observed risk reduction is due to bias, lifestyle, another co-factor or in fact a result of

RF EMF exposure is yet open. However, both the overall dependence of ORs on $\mathrm{N}_{\exp }$ and the dose-response analysis have one thing in common: they did not support alarming findings. Therefore, the synoptic analysis of the pooled epidemiologic results supports reassuring rather than alarming conclusions on health risks from mobile telephony.

\section{Conclusion}

The synoptic analysis proved to be a valuable tool and provided new insight into the data from epidemiologic studies on a potential link between telecommunication handset use and brain cancer. It exhibited that reported differences between published results of the SHG and AOS are reflected by two different data pools indicating major design differences in SHG's study and/or data analysis. In both data pools risk estimates clearly depend on the statistical power. However, it was shown that with increased number of exposed cases both data pools head towards the same OR value though from different sides. Existing uncertainties with regard to excessive and/or long-term handset use justify clarification by further research. Overall, the synoptic analysis supports reassuring rather than alarming conclusions on RF EMF health risks from mobile telephony.

\section{Conflict of Interest}

The author declares no conflict of interest.

\section{References}

[1] IARC (2002) IARC Monographs on the Evaluation of Carcinogenic Risks to Humans. Volume 80. Non-Ionizing Radiation, Part 1: Static and Extremely Low-Frequency (ELF) Electric and Magnetic Fields. IARC, Lyon.

[2] IARC (2011) IARC Monographs on the Evaluation of Carcinogenic Risks to Humans. Volume 102. Non-Ionizing Radiation, Part 2: Radio Frequency (RF) Electromagnetic Fields. IARC, Lyon.

[3] ICNIRP (1998) Guidelines for Limiting Exposure to Time-Varying Electric, Magnetic and Electromagnetic Fields (Up to $300 \mathrm{GHz})$. Health Physics, 74, 494-522.

[4] EU (2013) Directive on the Minimum Health and Safety Requirements Regarding Exposure of Workers to the Risks Arising from Physical Agents (Electromagnetic Fields). Official Journal of the European Union, L179, 1-18.

[5] IEEE (2005) IEEE Standard for Safety Levels with Respect to Human Exposure to Radio Frequency Electromagnetic Fields (Up to $300 \mathrm{GHz}$ ).

[6] Leitgeb, N. (2014) Childhood Leukemia Not Linked with ELF Magnetic Fields. Journal of Electric and Magnetic 
Analysis and Applications, 6, 174-183.

[7] Hardell, L. and Carlberg, M. (2013) Using the Hill Viewpoints from 1965 for Evaluating Strengths of Evidence of the Risk for Brain Tumors Associated with the Use of Mobile and Cordless Phones. Reviews on Environmental Health, 28, 97-106. http://dx.doi.org/10.1515/reveh-2013-0006

[8] Hardell, L., Carlberg, M., Söderqvist, F. and Hansson Mild, K. (2013) Case-Control Study of the Association between Malignant Brain Tumors between 2007 and 2009 and Mobile and Cordless Phone Use. International Journal of Oncology, 43, 1833-1845.

[9] Hardell, L., Carlberg, M., Söderqvist, F. and Hansson Mild, K. (2013) Pooled Analysis on Acoustic Neuroma Diagnosed 1997-2003 and 2007-2009 and Use of Mobile and Cordless Phones. International Journal of Oncology, 43, 1036-1044.

[10] Carlberg, M., Hansson Mild, K. and Hardell, L. (2013) Use of Mobile Phones and Cordless Phones Is Associated with Increased Risk for Glioma and Acoustic Neuroma. Pathophysiology, 20, 85-110. http://dx.doi.org/10.1016/j.pathophys.2012.11.001

[11] Carlberg, M., Söderqvist, F., Hansson Mild, K. and Hardell, L. (2013) Meningioma Patients Diagnosed 2007-2009 and the Association with the Use of Mobile and Cordless Phones: A Case-Control Study. Environmental Health, 12, 60. http://www.ehjournal.net/content/12/1/60 http://dx.doi.org/10.1186/1476-069X-12-60

[12] Hardell, L., Carlberg, M. and Hansson Mild, K. (2011) Pooled Analysis of Case-Control Studies on Malignant Brain Tumors and the Use of Mobile and Cordless Phones and Deceased Subjects. International Journal of Oncology, 38, 1465-1474. http://dx.doi.org/10.3892/ijo.2011.947

[13] Hardell, L., Carlberg, M. and Hansson Mild, K. (2006) Pooled Analysis of Two Case-Control Studies on Use of Cellular and Cordless Telephones and the Risk for Malignant Brain Tumors Diagnosed in 1997-2003. International Archives of Occupational and Environmental Health, 79, 630-639.

[14] Hardell, L., Carlberg, M. and Hansson Mild, K. (2005) Use of Cellular Telephones and Brain Tumor Risk in Urban and Rural Areas. Occupational and Environmental Medicine, 62, 390-394. http://dx.doi.org/10.1136/oem.2004.017434

[15] Interphone Study Group (2011) Acoustic Neuroma Risk in Relation to Mobile Telephone Use: Results of the INTERPHONE International Case-Control Study. Cancer Epidemiology, 35, 453-464.

[16] Interphone Study Group (2010) Brain Tumor Risk in Relation to Mobile Telephone Use: Results of the INTERPHONE International Case-Control Study. International Journal of Epidemiology, 39, 675-694. http://dx.doi.org/10.1093/ije/dyq079

[17] Moon, I.S., Kim, B.G., Kim, J., Lee, J.D. and Lee, W.S. (2014) Association between Vestibular Schwannomas and Mobile Phone Use. Tumor Biology, 35, 581-587. http://dx.doi.org/10.1007/s13277-013-1081-8

[18] Petterson, D., Mathiesen, T., Prochazka, M., Bergenheim, T., Florentzson, R., Harder, H., Nyberg, G., Siesjö, P. and Feychting, M. (2014) Long-Term Mobile Phone Use and Acoustic Neuroma Risk. Epidemiology, 25, 233-241. http://dx.doi.org/10.1097/EDE.0000000000000058

[19] Sato, Y., Akiba, S., Kubo, O. and Yamaguchi, N. (2011) A Case-Case Study of Mobile Phone Use and Acoustic Neuroma Risk in Japan. Bioelectromagnetics, 32, 85-93. http://dx.doi.org/10.1002/bem.20616

[20] Swerdlow, A.J., Feychting, M., Green, A.C., Kheifets, L. and Savitz, D. (2011) Mobile Phones, Brain Tumors and the Interphone Study: Where Are We Now? Environmental Health Perspectives, 119, 1534-1538. http://dx.doi.org/10.1289/ehp.1103693

[21] Lahkola, A., Salminen, T., Raitanen, J., Heinävaara, S., Schoemaker, M.J., Christensen, C.H., Feychting, M., Hohansen, C., Klaeboe, L., Lönn, S., Swerdlow, A.J., Tynes, T. and Auvinen, A. (2008) Meningioma and Mobile Phone Use-A Collaborative Case-Control Study in Five North European Countries. International Journal of Epidemiology, 37, 1304-1313. http://dx.doi.org/10.1093/ije/dyn155

[22] Takebayashi, T., Varsier, N., Kikuchi, Y., Wake, K., Taki, M., Watanabe, S., Akiba, S. and Yamaguchi, N. (2008) Mobile Phone Use, Exposure to Radiofrequency Electromagnetic Field, and Brain Tumor: A Case-Control Study. British Journal of Cancer, 98, 652-659. http://dx.doi.org/10.1038/sj.bjc.6604214

[23] Hours, M., Bernardt, M., Montestrucq, L., Arslan, M., Bergerer, A., Deltour, I. and Cardis, E. (2007) Cell Phones and Risk of Brain and Acoustic Nerve Tumors: The French INTERPHONE Case-Control Study. Epidemiol Public Health, 55, 321-332.

[24] Sadetzki, S., Chetrit, A., Jarus-Hakak, A., Cardis, E., Deutch, Y., Duvdevani, S., Zultan, A., Novikov, I., Freedman, L. and Wolf, M. (2007) Cellular Phone Use and Risk of Benign and Malignant Parotid Gland Tumors-A Nationwide Case-Control Study. American Journal of Epidemiology, 167, 457-467.

[25] Schlehofer, B., Schlaefer, K., Blettner, M., Berg, G., Böhler, E., Hetinger, I., Kunna-Grass, K., Wahrendorf, J. and Schüz, J. (2007) Environmental Risk Factors for Sporadic Acoustic Neuroma (Interphone Study Group Germany). Eu- 
ropean Journal of Cancer, 43, 1741-1747. http://dx.doi.org/10.1016/j.ejca.2007.05.008

[26] Hepworth, S.J., Schoemaker, M.J., Muir, K.R., Swerdlow, A.J., van Tongeren, M.J. and McKinney, P.A. (2006) Mobile Phone Use and Risk of Glioma in Adults: Case-Control Study. British Medical Journal, 332, 883. http://dx.doi.org/10.1136/bmj.38720.687975.55

[27] Klaeboe, L., Blaasaas, K.G. and Tynes, T. (2006) Use of Mobile Phones in Norway and Risk of Intracranial Tumors. European Journal of Cancer Prevention, 16, 158-164.

[28] Lahkola, A., Auvinen, A., Raitanen, J., Schoemaker, M.J., Christensen, H.C., Feychting, M., Johansen, C., Klaeboe, L., Lönn, S., Swerdlow, A.J., Tynes, T. and Salminen, T. (2006) Mobile Phones Use and Risk of Glioma in 5 North European Countries. International Journal of Cancer, 120, 1769-1775.

[29] Lönn, S., Ahlbom, A., Christensen, H.C., Johansen, C., Schüz, J., Edström, S., Henriksson, G., Lundgren, J., Wennerberg, J. and Feychting, M. (2006) Mobile Phone Use and Risk of Parotid Gland Tumor. American Journal of Epidemiology, 164, 637-643. http://dx.doi.org/10.1093/aje/kwj242

[30] Schüz, J., Böhler, E., Berg, G., Schlehofer, B., Hettinger, I., Schlaefer, K., Wahrendorf, J., Kunna-Grass, K. and Blettner, M. (2006) Cellular Phone, Cordless Phones and the Risk of Glioma and Meningioma (Interphone Study Group Germany). American Journal of Epidemiology, 163, 512-520.

[31] Lönn, S., Ahlbom, A., Hall, P. and Feychting, M. (2005) Long-Term Mobile Phone Use and Brain Tumor Risk. American Journal of Epidemiology, 161, 526-535. http://dx.doi.org/10.1093/aje/kwi091

[32] Schoemaker, M.J., Swerdlow, A.J., Ahlbom, A., Auvinen, A., Blaassas, K.G., Cardis, E., Christensen, H.C., Feychting, M., Hepworth, S.J., Johansen, C., Klaeboe, L., Lönn, S., McKinney, P.A., Muir, K., Raitanen, J., Salminen, T., Thomsen, J. and Tynes, T. (2005) Mobile Phone Use and Risk of Acoustic Neuroma: Results of the Interphone Case-Control Study in Five North European Countries. British Journal of Cancer, 93, 842-848. http://dx.doi.org/10.1038/sj.bjc.6602764

[33] Lönn, S., Hall, P. and Feychting, M. (2004) Mobile Phone Use and the Risk of Acoustic Neuroma. Epidemiology, 15, 653-659. http://dx.doi.org/10.1097/01.ede.0000142519.00772.bf

[34] Christensen, H.C., Schüz, J., Kosteljanetz, M., Paulsen, H.S., Thomsen, J. and Johansen, C. (2004) Cellular Telephone Use and Risk of Acoustic Neuroma. American Journal of Epidemiology, 159, 277-283. http://dx.doi.org/10.1093/aje/kwh032

[35] Auvinen, A., Hietanen, M., Luukkonen, R. and Koskela, R.S. (2002) Brain Tumors and Salivary Gland Cancers among Cellular Telephone Users. Epidemiology, 13, 356-359. http://dx.doi.org/10.1097/00001648-200205000-00018

[36] Inskip, P.D., Tarone, R., Hatch, E., Wilcosky, T.C., Sjapiro, W.R., Selker, R.G., Fine, H.A., Black, P.M., Loeffler, J.S. and Kinet, M.S. (2001) Cellular Telephone Use and Brain Tumors. New England Journal of Medicine, 344, 79-86. http://dx.doi.org/10.1056/NEJM200101113440201

[37] Ahlbom, A., Feychting, M., Green, A., Kheifets, L., Savitz, D.A. and Swerdlow, A.J. (2009) Epidemiologic Evidence on Mobile Phone and Tumor Risk: A Review. Epidemiology, 20, 639-652. http://dx.doi.org/10.1097/EDE.0b013e3181b0927d

[38] Kundi, M. (2009) The Controversy about a Possible Relationship between Mobile Phone Use and Cancer. Environmental Health Perspectives, 117, 316-324. http://dx.doi.org/10.1289/ehp.11902 